\title{
RADARSAT ScanSAR Roll Angle Estimation
}

\author{
D.C. Bast and I.G. Cumming \\ Radar Remote Sensing Group, Department of Electrical and Computer Engineering, \\ University of British Columbia, 2356 Main Mall, Vancouver, BC, Canada, V6T 1Z4 \\ E-Mail: dannob@telus.net, ianc@ece.ubc.ca
}

\begin{abstract}
Wide-swath SAR imagery obtained by the RADARSAT ScanSAR mode can suffer from radiometric artifacts. These artifacts arise from improper application of Range Dependent Gain Corrections (RDGCs), mainly due to insufficient knowledge of the satellite's roll angle. Specifically, roll angle estimation errors as small as 0.1 degrees can cause noticeable gain errors of $1 \mathrm{~dB}$ or more. Beam-stitching techniques exist which can reduce, but not eliminate, these errors in the beam overlap region

Current roll angle estimation algorithms do not consistently provide adequate results. These algorithms are susceptible to RDGC uncertainties in terms of pattern shape and gain offsets. This paper proposes a new data acquisition method, in which signal data is obtained during the beam switchover by transmitting pulses through one beam and receiving them with another beam. This "2-beam data" is then used in a modified algorithm to provide a more accurate and robust roll estimate. The logistics of acquiring 2-beam data are also explored. The effects of various roll angle estimation errors on different beam combinations are simulated. The algorithm results from a current and two proposed algorithms are compared. Algorithms using this 2-beam data can tolerate an overall lower mean scene $\sigma^{\circ}$ and more RDGC uncertainty than standard data.
\end{abstract}

Key words: ScanSAR, banding, calibration, roll angle.

\section{INTRODUCTION}

RADARSAT provides wide-swath coverage by using a scanning strategy that employs multiple antenna beams. During scene processing, the data collected by each beam must be stitched together to form a final scene. These data, however, have a range gain profile that must be corrected by the application of inverse beam-patterns, known as a Range Dependent Gain Corrections (RDGCs). Each beam has a unique RDGC that is defined in terms of gain versus elevation angle and is provided via the satellite payload file. Errors as large as $0.3^{\circ}$ in satellite roll angles have been observed [1]. These errors cause improper RDGC application, which results in gain variations in the image, creating a banding effect. Figure 1 illustrates a banding example.

\section{A. Beam-Stitching}

Beam stitching techniques exist which can reduce gain variations; however, gain variations and possible discontinuities will remain in the final scene if the

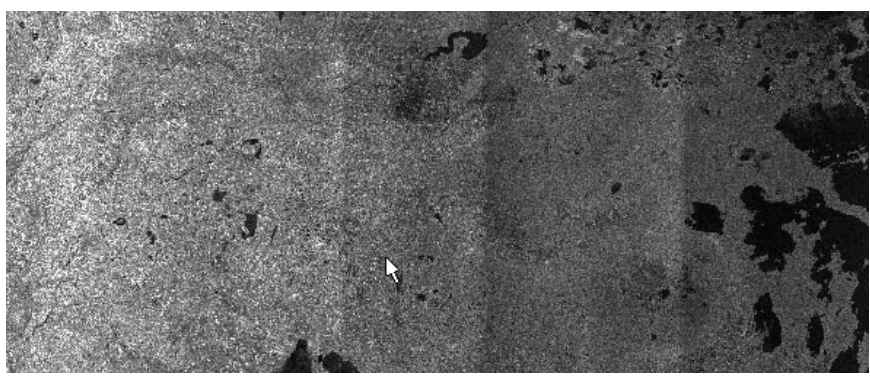

Figure 1: RADARSAT ScanSAR Wide B with banding. (C)SA

estimated roll angle is incorrect. The simplest stitching method is an abrupt transition from one beam to the next at a point where the beam-patterns are expected to have equal gain. This usually results in a gain discontinuity at the transition point, as in Fig. 1.

Another stitching technique merges the data from the two beams, using a linear weighting through a portion of the overlap region. Both methods are shown in Fig. 2. The linear merge will eliminate the gain discontinuity in the merge region; however, gain variations will remain and can be quite noticeable. It was determined that discontinuities as small as $0.2 \mathrm{~dB}$, and variations as small as $0.4 \mathrm{~dB}$, are noticed by the human eye.

\section{B. Current Roll Angle Estimation Algorithms}

The Canadian Data Processing Facility (CDPF) does not implement an automated ScanSAR roll angle
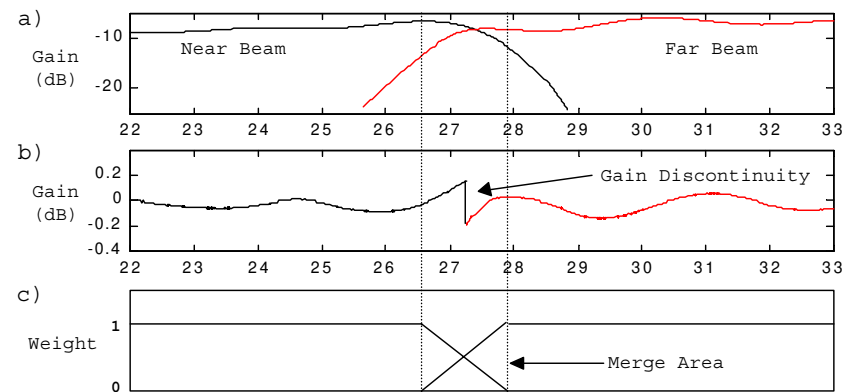

d)

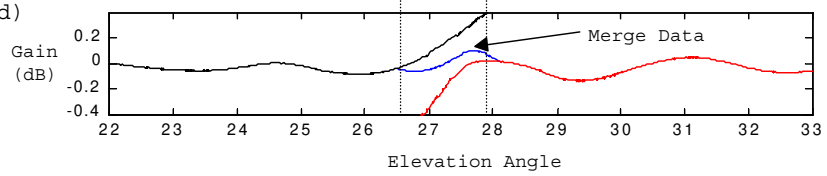

Figure 2: Implementation of two possible stitching methods: a) standard beam-patterns, b) beam patterns corrected with the RDGC, and stitched together, c) linear merge weighting, and d) gain variations after the linear merge 
estimator. All compensation for gain errors remaining after beam stitching is performed in the post-processing stage. Luscombe [2] was the first to suggest comparing data from the overlap region between beams to provide a roll estimate. Bamler [3] furthered the idea through use of a linear fit to the corrected data. Goulding [4] modified this idea and implemented a method which drove the mean of a log ratio of the corrected data as close as possible to a value of one. Jin [5] suggested a different approach by convolution of a kernel derived from the antenna pattern derivatives with the beam data. Dragosevic [6] expanded on Jin's algorithm and proposed an algorithm which simultaneously worked on all beams and incorporated gain offsets into the beams. Currently, none of these algorithms have been shown to provide consistent accuracy with RADARSAT-1 ScanSAR data.

\section{NEW DATA ACQUISITON METHOD}

ScanSAR operates by imaging approximately 60 to 120 range lines for each specific beam in blocks known as bursts. The satellite begins transmission of radar pulses at the beginning of each burst and ends pulse transmission several lines before the end of the burst. This results in unused range lines which can be used to receive pulses transmitted through one beam and received by another. This is achieved by keeping the transmitter on during the beam switchover period. The data, termed "2-beam data", received by this method has a range profile based on both the near and far beam patterns. The resulting new beam pattern is termed a "2-beam pattern".

\section{A. 2-Beam Patterns}

RADARSAT-1 ScanSAR has four beam modes which make use of five different beam combinations. Fig. 3 illustrates two of these beam pattern combinations along with their respective 2-beam pattern. The 2beam pattern is the average of the two normal beam patterns.

\section{B. Timing Considerations}

Transmit and receive timing parameter determine where the 2-beam data is recorded in signal memory. Range gate delays (RGDs) are used to position a receive window to receive data for a minimum and maximum slant range for each burst. RGDs differ for each beam and their difference will initially shift the 2-beam data a corresponding amount in signal memory. A PRF difference between beams will determine the size and direction of the range walk from the initial RGD shift. The result is that the receive window must be adjusted in order to receive 2-beam data. The simplest solution is to incorporate a single delay prior to the first transmit pulse of the upcoming burst such that the 2-beam data is received in the receive window. Fig. 4 illustrates a

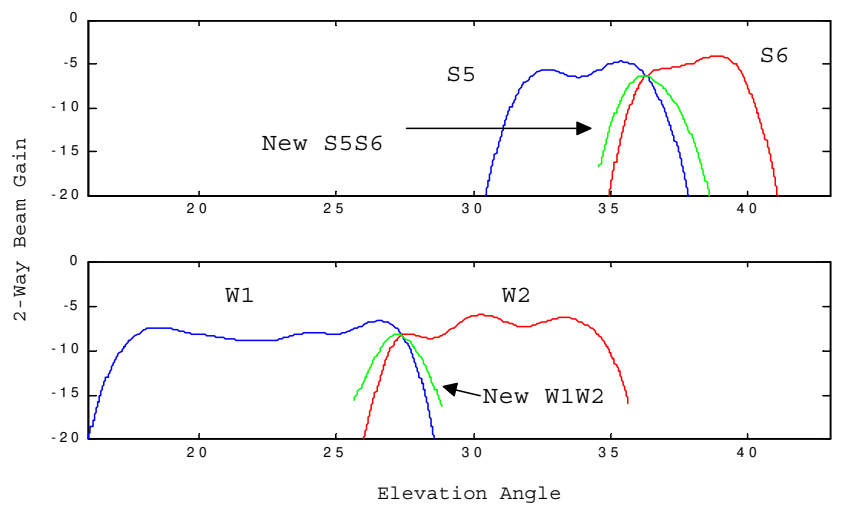

Figure 3: Two out of five possible RADARSAT-1 beam combinations.

typical range walk for 2-beam data with an appropriate delay in the start of the upcoming burst.

\section{PROPOSED ALGORITHMS}

Several types of algorithms were implemented to take advantage of the 2-beam data acquisition. After numerous trials, two algorithms provided the most consistent results. The first is a peak detection method and the second is a modified version of Goulding's algorithm, called the 2-beam method.

\section{A. Peak Detection Algorithm}

The 2-beam patterns have a well-defined peak and narrower width than normal beam patterns. Thus, a simple algorithm is proposed which fits a $2 \mathrm{nd}$, 3rd, and 4 th order polynomial to the average power present in several 2-beam data lines. The peak of each polynomial is assigned a weight, based on mean derivative of the difference of the 2-beam data and the polynomial. The weighted average peak location is compared against a peak look-up table, as calculated from the payload files, and a roll estimate is produced.

\section{B. 2-Beam Algorithm}

The 2-beam algorithm modifies Goulding's algorithm to include the 2-beam data. The algorithm begins by correcting the data (in $\mathrm{dB}$ ) from the near, far, and 2-beam patterns with their respective RDGC for a

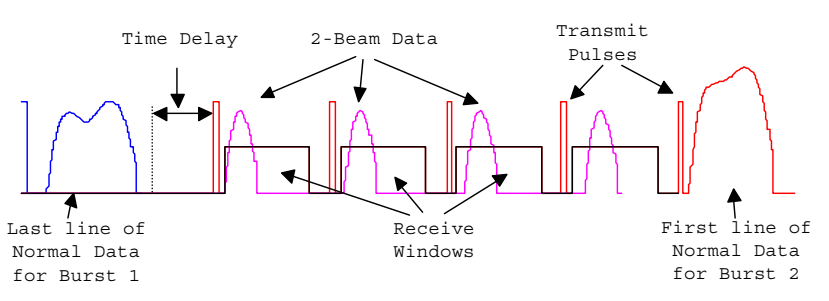

Figure 4: 2-Beam data is received and stored in signal memory according to differences in the PRF and RGD. A time delay at the start of the upcoming burst optimizes reception. 
specific roll angle. A ratio of the data between each of the beams is then made, on a point by point basis, resulting in three lines of ratios. A polynomial is fitted to each line so that small variations are not cancelled by random noise terms. The difference between these polynomials is then summed to produce a single value for this roll angle. The RDGCs are then shifted a small amount and the process is repeated. The sums for each case give an approximately linear result with respect to roll angle. Curve fitting is then used to find the zero-crossing. This process can be performed independently for each beam in the ScanSAR mode.

\section{Simulations}

Since 2-beam data is not currently acquired by RADARSAT-1, simulations were required to create ScanSAR data to test these algorithms. The simulations incorporated beam pattern uncertainties, various types of scene content, various levels of the mean scene $\sigma^{\circ}$ or $\bar{\sigma}^{\circ}$, and all the ScanSAR beam combinations. Several scenes were simulated and each of the proposed algorithms were implemented on the data. Goulding's algorithm was chosen for comparison since it was quite similar to the proposed 2-beam algorithm.

Each beam combination was analyzed using both the abrupt transition and linear merge stitching methods. A roll accuracy requirement was found for each beam combination and stitching method. These requirements are given in Table 1.

\begin{tabular}{|l|l|l|c|c|c|}
\hline & W1W2 & W2W3 & W2S5 & W3S7 & S5S6 \\
\hline A) & $0.034^{\circ}$ & $0.041^{\circ}$ & $0.035^{\circ}$ & $0.043^{\circ}$ & $0.028^{\circ}$ \\
\hline B) & $0.073^{\circ}$ & $0.077^{\circ}$ & $0.10^{\circ}$ & $0.135^{\circ}$ & $0.045^{\circ}$ \\
\hline
\end{tabular}

Table 1: Roll Angle Accuracy Requirements for the A) abrupt transition (with a $0.2 \mathrm{~dB}$ radiometric requirement), and $\mathrm{B}$ ) linear merge stitching method (with a $0.4 \mathrm{~dB}$ radiometric requirement)

\section{RESUltS}

The peak detection method was found to be useful for coarse roll angle estimation, which could reduce the search space for the more accurate 2-beam algorithm. The average accuracy of the peak detection method was approximately $0.165^{\circ}$ and showed considerable dependence on scene content and beam combination. For most of the simulations, the peak detection did not meet the requirements set out in Table 1.

However, the 2-beam algorithm did meet the requirements set by Table 1 . It could generally tolerate
3-4 $\mathrm{dB}$ lower $\bar{\sigma}^{\circ}$ and $0.10 \mathrm{~dB}$ more uncertainty in the beam pattern gain than Goulding's algorithm. The overall standard deviation was $0.15^{\circ}, 0.05^{\circ}$, and $0.05^{\circ}$ for the peak detection, 2-beam and Goulding algorithm, respectively.

\section{CONCLUSIONS}

A new ScanSAR data acquisition technique has been proposed which acquires signal data transmitted through one antenna beam pattern and received by another. Two proposed algorithms and one current algorithm were implemented using simulated 2-beam data and their results were compared. The new data and algorithms offer significant potential for improving roll estimates. The results suggest that the 2-beam algorithm can tolerate a lower scene $\bar{\sigma}^{\circ}$ and more beam pattern uncertainty than a current similar algorithm while still meeting radiometric requirements.

\section{ACKNOWLEDGEMENTS}

The authors wish to thank the following: RADARSAT Int. and CSA for providing images and data, Cathy Vigneron, Martie Goulding, and Tony Luscombe at MacDonald Dettwiler for their advice and assistance, and Bob Hawkins at CCRS for reviewing the research and providing advice and insight.

\section{REFERENCES}

[1] Hawkins R. K., Wolfe J., Murnaghan K., And Jefferies W. C. "Exploring the elevation beam overlap region in RADARSAT-1 ScanSAR", Proceedings of the CEOS Calibration Workshop, Tokyo, 2001

[2] Luscombe, A.P., "Using the overlap regions to improve ScanSAR calibration", CEOS SAR Calibration Workshop, ESA/ESTEC, Noordwijk, The Netherlands: ESA, 341346,1993

[3] Bamler, R., "Roll angle estimation in SIR-C ScanSAR processing", JPL Interoffice Memo 3340-94-030, 1994, unpublished

[4] Goulding, M., "Roll angle estimation for ScanSAR processing in the CDPF", MacDonald Dettwiler internal document, 1997, unpublished

[5] Jin, M. "Optimal range and doppler centroid estimation for a ScanSAR system", IEEE Trans. On Geoscience and Remote Sensing, vol.34, 479-488, 1996

[6] Dragosevic, M.V., "Roll angle measurement and compensation strategy for RADARSAT ScanSAR", CEOS SAR Workshop, ESC-CNES, Toulose, 1999 